\title{
Thrombopénie immunologique associée à l'infection HIV
}

Le mécanisme immunologique conduisant à la destruction périphérique des plaquettes dans la thrombopénie associée à l'infection HIV demeure imprécis : certaines données plaident en faveur de l'intervention d'auto-anticorps dirigés contre certains constituants plaquettaires, et d'autres pour le dépôt de complexes immuns sur les plaquettes. Bien qu'aucune protéine virale n'ait été décelée dans ces complexes immuns ou à la surface des plaquettes, le HIV paraît jouer un rôle réel, direct ou indirect, dans la physiopathogénie de la thrombopénie. En faveur de cette hypothèse, plaident sa place particulière tout au long de l'histoire naturelle de l'infection HIV et surtout la fréquente efficacité d'un traitement antiviral par la zidovudine (ou AZT).

\section{Éric Oksenhendler Maxime Seligmann}

E. Oksenhendler: chef de clinique-assistant. M. Seligmann: professeur. Service d'immunopathologie et d'hématologie, Inserm U.108, hôpital Saint-Louis, l, avenue ClaudeVellefaux, 75010 Paris, France. 'infection par le virus HIV s'accompagne d'une intense stimulation et prolifération polyclonale des cellules $\mathrm{B}[\mathrm{l}]$, responsable, chez plus de la moitié des patients asymptomatiques ou présentant un syndrome lymphadénopathique, d'une hypergammaglobulinémie [2]. Toutefois, si certains auto-anticorps ont une fréquence relativement élevée chez ces sujets, ils n'ont que très rarement une expression clinique. La thrombopénie immunologique occupe ainsi une place particulière car sa fréquence est évaluée à 10 à $15 \%$ chez les patients infectés par le virus HIV mais ne présentant pas de SIDA. Elle suscite diverses hypothèses physiopathogé- niques, mais son mécanisme n'est pas encore élucidé.

\section{Présentation clinique, hématologique et immunologique}

La présentation clinique et hématologique est tout à fait superposable à celle des purpura thrombopéniques immunologiques (PTI) chroniques de l'adulte. Le diagnostic peut être porté sur un hémogramme systématique ou devant l'existence de signes hémorragiques cutanés et/ou muqueux qui restent le plus souvent sans gravité. Les accidents hémorragiques viscéraux graves, qu'ils soient digestifs, rétiniens ou cérébroméningés, sont rares. Le myélo- 
gramme est riche en mégacaryocytes, et l'étude isotopique, lorsqu'elle est pratiquée, montre une destruction périphérique rapide, et exclusivement splénique dans $85 \%$ des cas [3]. Le taux des immunoglobulines liées aux plaquettes est élevé chez 76 à $94 \%$ des malades, en fonction des techniques utilisées. La recherche dans le sérum d'anticorps anti-plaquettes n'est positive que dans 29 à $61 \%$ des cas [4-6]. L'ensemble de ces données confirme le caractère périphérique et immunologique de la thrombopénie.

La survenue d'une thrombopénie immunologique a été signalée chez des patients appartenant à tous les groupes considérés comme «à risque» pour le SIDA : homosexuels, toxicomanes et hémophiles, puis chez les patients contaminés par transfusion ou par voie hétérosexuelle. Les données épidémiologiques, immunologiques et virologiques ont permis de rattacher cette complication à l'infection par le virus HIV.

Chez environ la moitié des malades, la thrombopénie est associée à d'autres signes cliniques: polyadénopathie chronique (15 à $57 \%$ ), splénomégalie (8 à $17 \%$ ), amaigrissement, diarrhée chronique ou candidose buccale (10 à $17 \%$ ). Toutefois, chez $50 \%$ des patients, la thrombopénie représente la seule manifestation de l'infection HIV [6-8]. Parmi les patients suivis par notre groupe, $36 \%$ ont une lymphopénie importante avec un déficit net en lymphocytes CD4 $\left(<0,4 \times 10^{9} / 1\right)$ au moment du diagnostic de purpura thrombopénique. La recherche de l'antigénémie p25 s'est révélée initialement positive chez 11 des 75 patients testés (15\%). La fréquence d'autres anomalies immunologiques ne semble pas différente de celle observée chez les patients séropositifs non thrombopéniques et, par ailleurs, asymptomatiques ou présentant un syndrome lymphadénopathique: une hypergammaglobulinémie polyclonale supérieure à $20 \mathrm{~g} / \mathrm{l}$ est notée chez $50 \%$ d'entre eux, et une immunoglobuline monoclonale est présente dans $7 \%$ des cas ; le test de Coombs est parfois positif (10\%), mais n'est qu'exceptionnellement associé à une anémie hémolytique auto-immune ; des anticorps anti-nucléaires sont $\mathrm{m} / \mathrm{s} n^{\circ} 4$ vol. 5 , avril 89 retrouvés à un taux faible chez $6 \%$ des malades, et des anticorps anticardiolipides chez $14 \%$.

\section{Place de la thrombopénie dans I'histoire naturelle de l'infection HIV}

La thrombopénie peut être présente à n'importe quelle étape de l'histoire naturelle de l'infection HIV, toutefois sa grande fréquence $(\simeq 50 \%)$ chez les malades atteints de SIDA, recouvre probablement l'intrication de plusieurs mécanismes et/ou étiologies, en particulier infectieuses et toxiques.

En dehors du SIDA, elle survient, avec une fréquence de 5 à $15 \%$, à tous les stades qui marquent la progression du déficit immunitaire. Dans notre expérience, si l'on se réfère à la classification du $\mathrm{CDC}^{*}, 53 \%$ de nos patients appartiennent au groupe II, $30 \%$ au groupe III, et $17 \%$ au groupe IV. Dans certaines circonstances "privilégiées», la date de séroconversion a pu être cernée avec précision, permettant d'en déduire le délai d'apparition de la thrombopénie; ainsi, dans un groupe de 27 hémophiles, ce délai est en moyenne de deux ans avec des extrêmes de 12 à 42 mois. La fréquence des signes généraux et des infections non opportunistes, ainsi que de la lymphopénie et du déficit en lymphocytes CD4 et une prévalance de $15 \%$ de l'antigénémie P25 au moment du diagnostic suggèrent que ces malades se situent souvent en aval du porteur asymptomatique dans l'histoire naturelle de la maladie. Toutefois, cette complication immuno-hématologique peut s'observer au cours des stades précoces, y compris la primo-infection HIV où la thrombopénie est le plus souvent modérée et transitoire [9]. Enfin, le

* Classification CDC: groupe I = primoinvasion; groupe II = asymptomatique groupe $I I I=$ syndrome lymphadénopathique; groupe $I V=$ infections ou tumeurs secondaires. purpura thrombopénique peut représenter la seule manifestation de l'infection HIV chez le nourrisson, après contamination néonatale [10]. La valeur pronostique intrinsèque de la thrombopénie est difficile à évaluer en l'absence d'études comparatives rigoureuses. Dans les études portant sur le suivi de patients présentant un syndrome lymphadénopathique, la thrombopénie paraît avoir une valeur pronostique péjorative [2, 11], alors que, dans une cohorte de 44 homosexuels avec un purpura thrombopénique suivis pendant une durée totale de 844 mois-personnes, la survie sans SIDA à trois ans, évaluée à $65 \%$, ne semble pas différente de celle observée chez les patients non thrombopéniques suivis dans la même ville [12]. Dans notre expérience, portant sur 132 patients suivis sur une durée totale de 2987 mois-patients, le taux de survie sans SIDA après quatre ans d'évolution de la thrombopénie est d'environ $78 \%$ (figure 1, p. 222).

L'ensemble de ces données suggère (comme pour les manifestations neurologiques) une physiopathogénie indépendante du déficit immunitaire mais liée, directement ou indirectement, à l'infection virale.

\section{Aspects} thérapeutiques

Les principaux traitements médicaux visent à réduire l'hyperdestruction des plaquettes. Les corticoïdes, utilisés par analogie avec le traitement du PTI chronique de l'adulte, permettent d'obtenir une réponse initiale chez environ $60 \%$ des malades. Toutefois, la correction de la thrombopénie ne persiste à l'arrêt du traitement que chez $15 \%$ des patients. Ces résultats peu encourageants, conjugués au risque potentiel d'aggravation du déficit immunitaire, n'incitent pas à retenir les corticoïdes comme traitement de première intention des thrombopénies immunologiques associées à l'infection HIV, La vincristine, la colchicine, le danazol ou l'interféron $\alpha$ ne peuvent guère jouer qu'un rôle d'appoint chez certains patients.

L'utilisation d'immunoglobulines polyvalentes à forte dose par voie intraveineuse permet, dans 75 à $80 \%$ des cas, une remontée rapide et 


\section{RÉFÉRENCES}

1. Lane C, Masur H, Edgard LC, et al. Abnormalities of B-cell activation and immunoregulation in patients with the acquired immunodeficiency syndrome. $N$ Engl J Med $1983 ; 309: 453-8$

2. Gold JWM, Weikel CS, Godbold J, et al. Unexplained persistent lymphadenopathy in homosexual men and the acquired immune deficiency syndrome. Medicine $1985 ; 64$ (3) : 203-13.

3. Bel-Ali Z, Dufour V, Najean Y. Platelet kinetics in human immunodeficiency virus induced thrombocytopenia. Am J Hematol $1987 ; 26: 299-304$.

4. Van der Lelie J, Lange JMA, Vos JJE, et al. Autoimmunity against blood cells in human immunodeficiency-virus (HIV) infection. Br J Haematol 1987; 67 : 109-14.

5. Murphy MF, Metcalfe $\mathrm{P}$, Waters $\mathrm{AH}$, et al. Incidence and mechanism of neutropenia and thrombocytopenia in patients with human immunodeficiency virus infection. $\mathrm{Br} \mathrm{J} \mathrm{Hae}$ matol $1987 ; 66: 337-40$

6. Oksenhendler E, Bierling P, Farcet JP, et al. Response to therapy in 37 patients with HIV-related thrombocytopenic purpura. $\mathrm{Br} J$ Haematol 1987 ; 66 : 491-5.

7. Abrams DI, Kiprov DD, Goebert JJ, et al. Antibodies to human T-lymphotropic virus type III and development of the acquired immunodeficiency syndrome in homosexual men presenting with immune thrombocytopenia. Ann Intern Med 1986; $104: 47-50$

8. Savona S, Nardi MA, Lennette ET, et al. Thrombocytopenic purpura in narcotics addicts. Ann Intern Med 1985; 102 (6) : 737 41.

9. Lima J, Ribera A, Garcia-Bragado F, et al. Antiplatelet antibodies in primary infection by human imunodeficiency virus. Ann Intern Med 1987 ; 106 (2) : 333.

10. Saulsbury FT, Boyle RJ, Wykoff RF, et al. Thrombocytopenia as the presenting manifestation of human T-lymphotropic virus type III infection in infants. $J$ Pediatr $1986 ; 109: 30-4$

11. Polk BF, Fox R, Brookmeyer R, et al. Predictors of the acquired immunodeficiency syndrome developping in a cohort of seropositive homosexual men. $N$ Engl J Med 1987 ;

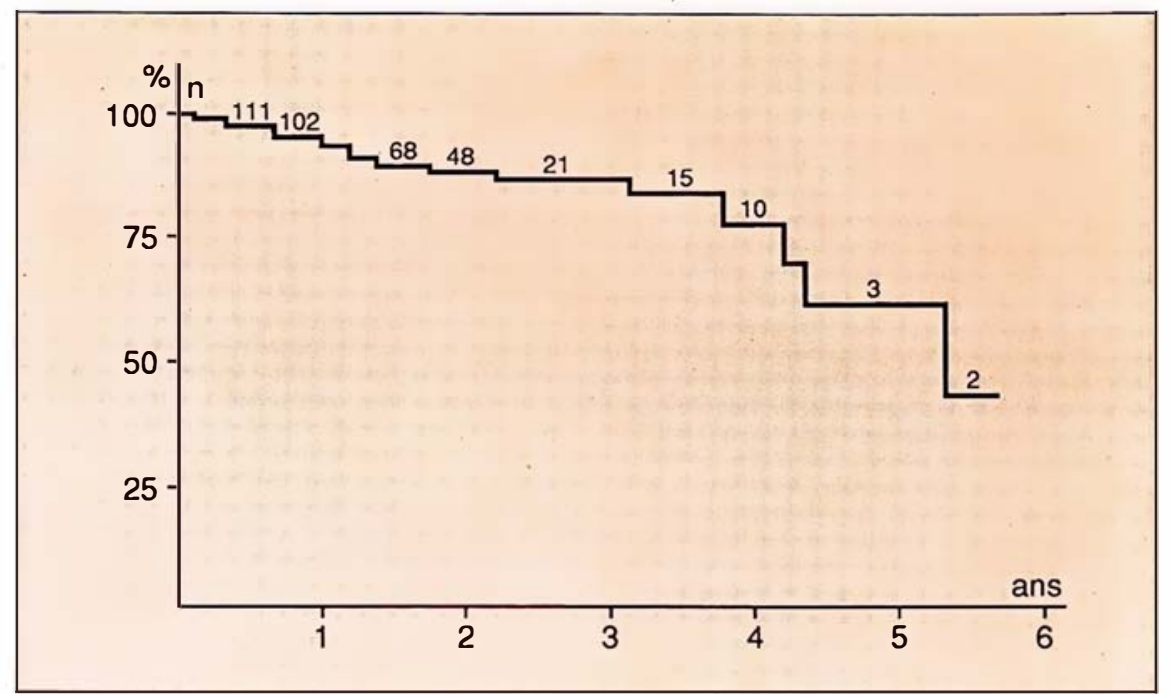

Figure 1. Thrombopénie immunologique associée à l'infection HIV: survie sans SIDA. $n=132$ (nombre de patients).

importante du chiffre de plaquettes. Cette réponse est le plus souvent transitoire. Les immunoglobulines anti-D $(25 \mu \mathrm{g} / \mathrm{kg} \times 2$ jours $)$ permettent d'obtenir, chez les malades Rhésus positif, une réponse comparable à celle obtenue avec les immunoglobulines polyvalentes, mais là encore transitoire. Cependant, le coût plus faible et le temps de perfusion beaucoup plus court permettent de réaliser un traitement d'entretien par perfusions itératives chez des malades ambulatoires [13]. Une interaction spécifique entre hématies du patient et anticorps anti-Rhésus injectés est nécessaire, excluant ainsi une action directe et non spécifique des antiD [14]. Le mécanisme d'action implique probablement une inhibition compétitive, au niveau des cellules phagocytaires, entre les plaquettes recouvertes d'immunoglobulines et les hématies sensibilisées par les antiD perfusées.

Deux études ont récemment montré l'efficacité de la zidovudine (AZT) chez des patients présentant un purpura thrombopénique associé à une infection HIV. L'une concerne dix patients traités alternativement par zidovudine et placebo[15]; l'autre, menée par notre groupe, porte sur 34 patients traités de façon prospective par la zidovudine pendant au moins quatre mois [16]. Trois des dix patients traités par $1000 \mathrm{mg} / \mathrm{j}$ et 12 des 24 patients recevant $1500 \mathrm{mg} / \mathrm{j}$ ont eu une ascension nette et persistante du chiffre des plaquettes. La tolérance hématologique a été très bonne et aucune modification de posologie n'a été nécessaire pendant les quatre premiers mois de traitement. Ce taux de $40 \%$ de réponses persistantes permet d'envisager l'utilisation de la zidovudine comme traitement de fond de la thrombopénie immunologique liée à l'infection HIV. Ce traitement est insuffisant pour les patients présentant des signes hémorragiques, car son délai d'action varie entre deux et dix semaines.

L'indication de la splénectomie se pose devant la persistance d'une thrombopénie profonde, symptomatique, résistant au traitement médical. La splénectomie est actuellement le seul traitement permettant, chez 75 à $90 \%$ des patients, une correction durable de la thrombopénie [6, 7, 17]. Le risque potentiel de l'aggravation du déficit immunitaire doit être pris 
en considération, bien qu'aucune étude n'ait actuellement mis en évidence une accélération de l'évolution vers le SIDA chez les patients splénectomisés [17, 18].

\section{Physiopathogénie de la thrombopénie}

La discussion du mécanisme responsable de la destruction périphérique des plaquettes se pose à deux niveaux : l'intervention d'auto-anticorps ou de complexes immuns et la responsabilité, directe ou indirecte, du HIV (figure 2).

L'implication d'auto-anticorps antiplaquettes a initialement été suggérée par Savona et al. qui, étudiant 70 toxicomanes, trouvaient, dans plus de $50 \%$ des cas, une fraction sérique IgG $7 \mathrm{~S}$ capable de se fixer par son fragment $F(a b ')_{2}$ sur des plaquettes normales [8]. Par la suite,
Van der Lelie et al. montrent que les éluats plaquettaires sont composés d'immunoglobulines reconnaissant les plaquettes de donneurs séronégatifs, mais non les plaquettes de sujets atteints de maladie de Glanzmann type I; cette constatation suggère ainsi une activité auto-anticorps dirigée contre un des épitopes du complexe glycoprotéique Gp IIb-IIIa, comme au cours de certains PTI chroniques [4]. Dans l'expérience de notre groupe (A. Bettaieb et P. Bierling), l'étude en immunoprécipitation des éluats obtenus chez 35 patients permet de retrouver six fois une activité anticorps dirigée contre une glycoprotéine majeure de la membrane plaquettaire (dont 2 anti-IIb et lanti-IIIa). Pour Stricker et al. [19], les auto-anticorps antiplaquettaires présents dans le sérum et les éluats reconnaîtraient également les plaquettes de throm-

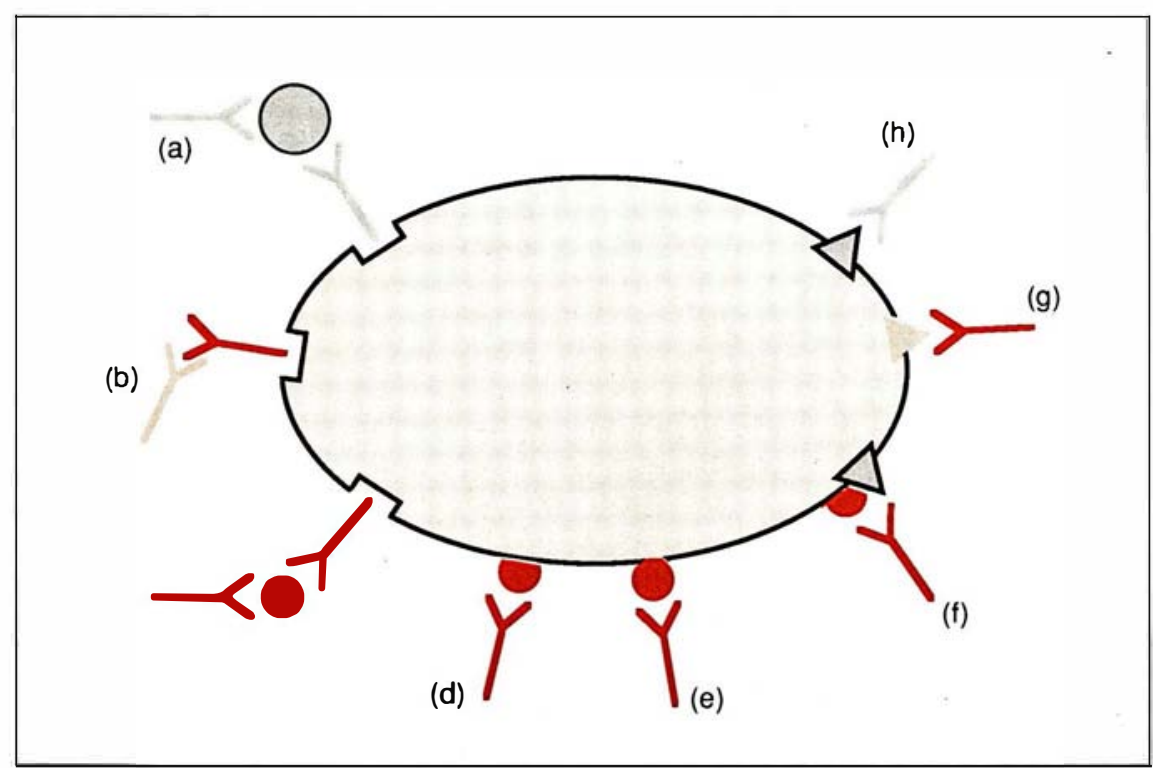

Figure 2. Thrombopénie immunologique associée à l'infection HIV hypothèses physiopathogéniques. $a=$ complexes immuns non spécifiques ; $b=$ complexes immuns : anti-HIV/anti-anti-HIV; $c=$ complexe immuns : HIV/anti$H I V ; d=H I V$ exprimé à la surface plaquettaire/anti-HIV; $e=H I V$ adsorbé à la surface plaquettaire/anti-HIV; $f=$ épitope résultant de la modification d'une glycoprotéine membranaire par une structure virale; $g=$ épitope partagé par glycoprotéine membranaire et par une protéine virale; $h=$ auto-anticorps antiglycoprotéine membranaire.

$m / s n^{\circ} 4$ vol. 5, avril 89

basthénie de Glanzmann, ainsi que celles du syndrome de Bernard Soulier, déficitaires en $\mathrm{Gp}$ I. La cible de ces auto-anticorps serait une protéine de $25 \mathrm{kDa}$ également présente sur cellules Vero, et dont la séquence $\mathrm{N}$-terminale ressemblerait à celle d'un précurseur de la polymérase du virus HIV [20]. Il faut noter que ces anticorps ne sont présents que chez les patients homosexuels et qu'ils sont également trouvés chez les sujets séropositifs mais non thrombopéniques. Leur analogie avec les anticorps anti-P25 du virus HIV reste très discutée.

La responsabilité de dépôts non spécifiques de complexes immuns et/ou de complément a d'abord été suggérée par Walsh et al. [21] qui, trouvent chez 29/33 patients homosexuels avec purpura thrombopénique, un taux de complexes immuns circulants trois à dix fois supérieur à celui observé au cours du PTI chronique de l'adulte, ou chez les homosexuels non thrombopéniques, ainsi que des quantités d'immunoglobulines et de complément liées aux plaquettes très élevées ( 10 à 20 fois supérieures aux valeurs normales, et plus de quatre fois supérieures aux valeurs observées au cours du PTI chronique). Les immunoglobulines obtenues par élution n'étaient pas capables de se fixer sur des plaquettes allogéniques $^{* *}$, et si une activité antiplaquettes était trouvée dans le sérum de certains patients, elle était absente de la fraction IgG isolée de ces mêmes sérums. La même équipe a ensuite montré que les éluats plaquettaires comportent des anticorps anti-idiotypiques ${ }^{* *}$ à activité anti-F $\left(\mathrm{ab}^{\prime}\right)_{2}$ [22] et pratiquement sont constitués, pour moitié, d'anticorps à activité anti-HIV [23]. Bien que la présence de déterminants viraux n'ait pas pu être démontrée au sein de ces complexes, la prépondérance de la spécificité anti-HIV des immunoglobulines présentes dans les éluats, plaide en faveur d'un mécanisme faisant

** C'est-à-dire de même groupe tissulaire. ** Un anticorps anti-idiotype est dirigé contre le déterminant de spécificité anticorps d'un autre type d'immunoglobuline; il est donc une "copie conformationnelle " de l'antigène... ici, d'antigènes viraux de HIV. 
12. Holzman RS, Walsh CM, Karpatkin S Risk of the acquired immunodeficiency syndrome among thrombocytopenic and non thrombocytopenic homosexual men seropositive for the human immunodeficiency virus. Ann Intern Med 1987 ; 106 : 383-6.

13. Oksenhendler E, Bierling P, Brossard Y, et al. Anti-Rh immunoglobulin therapy for human immunodeficiency virus-related immune thrombocytopenic purpura. Blood $1988 ; 5$ : 1499-502.

14. Bierling P, Oksenhendler E, Habibi B, et al. Anti-R hesus antibodies as treatment for HIV-related immune thrombocytopenia. Ann Intern Med 1987; 106 : 773-4

15. The Swiss group for clinical studies on the Acquired Immunodeficiency Syndrome (AIDS). Zidovudine for the treatment of thrombocytopenia associated with human immunodeficiency virus (HIV). Ann Intern Med 1988; 109 : 718-21.

16. Oksenhendler $\mathrm{E}$, Bierling $\mathrm{P}$, Ferchal $\mathrm{F}$, et al. Zidovudine for thrombocytopenic purpura related to human immunodeficiency virus (HIV) infection. Ann Intern Med 1989; 110 : 365-8.

17. Walsh C, Krigel R, Lennette E, et al. Thrombocytopenia in homosexual patients. Ann Intern Med 1985 ; 103 : 542-5.

18. Barbui $T$, Cortelazzo S, Minetii B et al. Does splenectomy enhance risk of AIDS in HIV-positive patients with chronic thrombocytopenia? Lancet $1987 ; 2: 342-3$

19. Stricker RB, Abrams DI, Corash L, et al. Target platelet antigen in homosexual men with immune thrombocytopenia. $N$ Engl Med 1985 ; 313 (22) : 1375-80.

20. Stricker RB, Grimaldi AG, Neymann PD. Resemblance between a target platelet antigen as a human immunodeficiency virus pre cursor protein. Blood 1988; 72 : 360 a (abstr 1350).

21. Walsh CM, Nardi MA, Karpatkin S. On the mechanism of thrombocytopenic purpura in sexually active homosexual men. $N$ Engl J Med 1984; 311 (10) : 635-9.

22. Yu JR, Lennette ET, Karpatkin S. Anti$\mathrm{F}\left(\mathrm{ab}^{\prime}\right)_{2}$ antibodies in thrombocytopenic patients at risk for acquired immunodeficiency syndrome. J Clin Invest 1986; 77 : 1756-61

23. Karpatkin S, Nardi M, Lennette ET, et al. Anti-human immunodeficiency virus type I antibody complexes on platelets of seropositive thrombocytopenic homosexuals and narcotics addicts. Proc Natl Acad Sci (USA) 1988 85 : 9763-7. intervenir des anticorps anti-HIV, vraisemblablement complexés à des anticorps anti-idiotypiques ***.

Il n'y a, à l'heure actuelle, aucun élément prouvant l'intervention directe du virus HIV, que ce soit par le biais de l'infection des précurseurs mégacaryocytaires ou par un mécanisme d'adsorption passive des protéines virales sur la membrane plaquettaire. Le virus HIV pourrait cependant intervenir dans la genèse d'auto-anticorps réagissant soit avec des épitopes communs à certaines glycoprotéines plaquettaires et à certaines protéines virales, soit avec de nouvelles structures antigéniques liées à la modification d'une glycoprotéine plaquettaire par association à un déterminant viral. De telles hypothèses permettraient de rendre compte de la présence conjointe, dans certains éluats plaquettaires, d'anticorps reconnaissant la Gp IIbIIIa et d'anticorps anti-HIV.

Quoi qu'il en soit, le virus HIV a très vraisemblablement un rôle indirect important, du fait de la stimulation polyclonale B qu'il induit. Cette stimulation entraîne une augmentation de la production de complexes immuns circulants, qu'ils soient spécifiques ou non du virus HIV, et peut favoriser l'émergence d'auto-anticorps.

Si le mécanisme immunologique exact conduisant à la destruction périphérique des plaquettes reste très discuté, la fréquence de la thrombopénie par rapport aux autres manifestations auto-immunes, sa place particulière tout au long de l'histoire naturelle de l'infection HIV, indépendamment de l'importance du déficit immunitaire, et l'efficacité d'un traitement antiviral par la zidovudine sont autant d'éléments qui plaident en faveur d'un rôle réel, direct ou indirect, du virus HIV dans la physiopathogénie de la thrombopénie

*** Un anticorps anti-idiotype est dirigé contre le déterminant de spécificité anticorps d'un autre type d'immunoglobuline; il est donc une "copie conformationnelle " de l'antigène... ici, d'antigènes viraux de HIV.

\section{Summary}

HIV-related thrombocytopenia

Immunologic thrombocytopenic purpura is frequent in HIV-infected individuals and occurs in all risk groups and at all stages of the natural history, from primo-invasion to full-blown AIDS. Corticosteroids, high dose intravenous immunoglobulin and anti-Rhesus immunoglobulin induce only a transient increase in platelet counts. Zidovudine was recently shown to provide a sustained response in about $40 \%$ of the patients. Splenectomy is effective in most cases with persistent, profound and symptomatic thrombocytopenia and should thus be performed in such instances in spite of its potential hazards in immunocompromized patients. The precise mechanism responsible for platelet destruction remains presently unknown. Whereas some data support the direct effect of auto-antibodies to platelet constituants, other findings argue in favour of deposition of immune complexes containing anti-HIV antibodies. Although viral components were detected neither in such antibody complexes nor on the platelet membrane, a direct or indirect role for HIV in the pathogenesis of thrombocytopenia should seriously be considered.

\section{TIRÉS A PART}

M. Seligmann. 